\title{
Point-contact-spectroscopy investigation of the Kondo size effect in CuCr and AuFe alloys
}

\author{
V. V. Fisun and I. K. Yanson \\ B. Verkin Institute for Low Temperature Physics and Engineering, National Academy of Sciences of Ukraine \\ 47, Lenin Ave. , 61164, Kharkov, Ukraine \\ E-mail: fisun@ilt.kharkov.ua \\ J. A. Mydosh and J. M. van Ruitenbeek \\ Kamerlingh Onnes Laboratorium, Leiden University, PO Box 9504, NL-2300 RA Leiden, The Netherlands
}

Received January 21, 2000

\begin{abstract}
Size effects in Kondo scattering are studied on $\mathrm{CuCr}$ and $\mathrm{AuFe}$ alloys $\left(T_{K}=2 \mathrm{~K}\right.$ and $0.2 \mathrm{~K}$, respectively) by applying point-contact spectroscopy in break-junction type contacts. It is shown that as the contact diameter is decreased under the condition of ballistic electron transport, the size effect enhances the interaction of the conduction electrons with the Kondo impurity ( as compared to the phonons) and increases the Kondo temperature in the contact region. In an external magnetic field the size effect decreases the negative magnetoresistance in $\mathrm{CuCr}$ and suppresses the Kondo peak splitting in $\mathrm{AuFe}$.
\end{abstract}

PACS: 72.15.Qm, 75.20.Hr

\section{Introduction}

Point-contact (PC) spectroscopy measurements of Kondo alloys performed on pressed contacts [1] revealed that the interaction between conduction electrons and magnetic impurities shows itself in the PC spectra as a peak at zero bias in the first derivative of the voltage-current $(V-I)$ curve $d V / d I(V)$. The energy region of the Kondo peak manifestation is bounded from above by the energy of phonon features of the noble metals in which these impurities are dissolved and lies in the range between 0 and approximately $10 \mathrm{mV}$. The Kondo peak intensity is sensitive both to the chemical species of the magnetic impurity that defines the Kondo temperature and to its concentration (at.\%) in a given alloy. For example, the PC spectra for $\mathrm{CuFe}(0.1$ at.\%) display a Kondo peak of high intensity with a pronounced smearing of the phonon characteristics, as opposed to the well-defined spectra of $\mathrm{CuMn}$ for the same concentration. A similar smearing of the phonon characteristics and an increase in the Kondo peak are observed with increasing impurity concentration in CuMn alloys as well.

The increase in the differential resistance in the vicinity of the Kondo peak follows approximately the logarithmic relation $d V / d I(V) \propto-\ln (V)$, also typical of the increase in the resistance of these alloys at low temperatures.

More recently, a CuMn Kondo alloys with different magnetic impurity contents were studied using break-junction contacts [2]. It was found that a decrease in the point contact diameter resulted in an enhancement of the interaction between conduction electrons and Kondo impurities as compared to the electron-phonon interaction (EPI) and in a considerable increase of the apparent Kondo temperature in the point contact area. Moreover, the decrease in the contact diameter resulted in a disappearance of the Kondo peak splitting induced by external magnetic field (see Fig. 7 in Ref. [2] (b)).

An explanation for the observed Kondo size effect was presented in a theoretical investigation of such systems [3] and may be induced by the fluctuation of a local electron density of states at the Fermi energy in a bridge-like point contact linking massive electrodes.

Size effects were also studied in measurements of the resistance of $\mathrm{CuCr}$ films as a function of their width at low temperatures, the film thickness remaining unchanged [4]. It was found that the Kondo resistance anomaly was suppressed in a film of width smaller then $10 \mu \mathrm{m}$. This length scale is 
consistent with an heuristic estimate of the radius of the spin-electron correlation. Note, that the sign of the size effect observed is opposite to that in PC measurements.

Investigations into size dependences of the resistivity and thermopower [5] performed on mesoscopic AuFe films and wires demonstrate that for thickness smaller than $300 \mathrm{~nm}$ the Kondo contribution of $\Delta \rho$ and $\delta S(T)$ is also suppressed, i. e. sign of the size effect is same as in above studied film. In addition, at high impurity concentrations spin-glass ordering destroys the size effect.

Resistive measurements of Kondo AuFe wires versus their widths were made in Ref. [6]. The Kondo resistance was found to be independent of reduction in wire width down to a wire thickness less than $38 \mathrm{~nm}$, much less than the Kondo correlation length $\xi_{K}=\hbar v_{F} / k_{B} T_{K} \approx 38 \mu \mathrm{m}$.

\section{Experimental}

In this paper we present PC spectroscopy data on the Kondo size effect in $\mathrm{CuCr}(0.1$ at.\%) and AuFe (0.1 at.\%) alloys with a Kondo temperature of 2 and $0.2 \mathrm{~K}$, respectively. Junctions that satisfy conditions for ballistic electron transport, where the junction diameter $d$ is much smaller than the elastic and inelastic mean free paths $l_{e}$ and $l_{i}$ (ballistic regime), were formed between massive electrodes by the break-junction technique [7]. This makes it possible to study the interaction of the conduction electrons in the contact area with phonons and magnetic impurities on the same constrictions of different diameters.

The Kondo alloys were prepared at the Kamerling Onnes laboratory. A massive sample was used to prepare a wire $0.2 \mathrm{~mm}$ thick. The use of a sample in the form of a small diameter wire permits notching by using a sharp knife at the site where we want it to break. This notch is produced at the middle of a 10 -mm-long wire section and reduces its diameter several fold. The sample with the notch was etched in a mixture of acids $(1 / 3$ acetic, $1 / 3$ orthophosphoric, and $1 / 3$ nitric acid for $\mathrm{CuCr}$ and aqua regia for AuFe) and annealed in high vacuum at $T=600^{\circ} \mathrm{C}$ for 2.5 hours followed by natural cooling down. The sample was fixed on a substrate from beryllium bronze coated with a kapton film, using Staycast epoxy. An important factor for PC resistance stability is that the glue droplets be placed as close as possible to the notch. The notch should be made at the middle of the substrate, which is $15 \mathrm{~mm}$ long in our measurements. The substrate with the glued sample is placed in a bending beam that permits the substrate middle part to be pressed from the side opposite to that carrying the sample, with the substrate edges remaining fixed. The control over bending is realized from the outside of the cryostat. The substrate deflection results in a smooth extension of the sample at the site of the notch and hence in a decrease of its diameter to required values. Four wires connect the sample with a measuring circuit. A temperature of $1.6 \mathrm{~K}$ is achieved in a liquid helium cryostat that contains a superconducting solenoid.

The first $d V / d I(V)$ and the second $d^{2} V / d I^{2}(V)$ derivatives of the current-voltage curves were measured by a standard modulation technique with the use of lock-in amplifiers. The Kondo peak was measured by using a bridge circuit to produce higher spectral resolution for lower modulation.

\section{Results}

Typical spectra of the first $d V / d I(V)$ and the second $d^{2} V / d I^{2}(V)$ derivatives of the $V$-I curves for $\mathrm{CuCr}(0.1$ at.\%) point contacts are shown in Fig. 1.
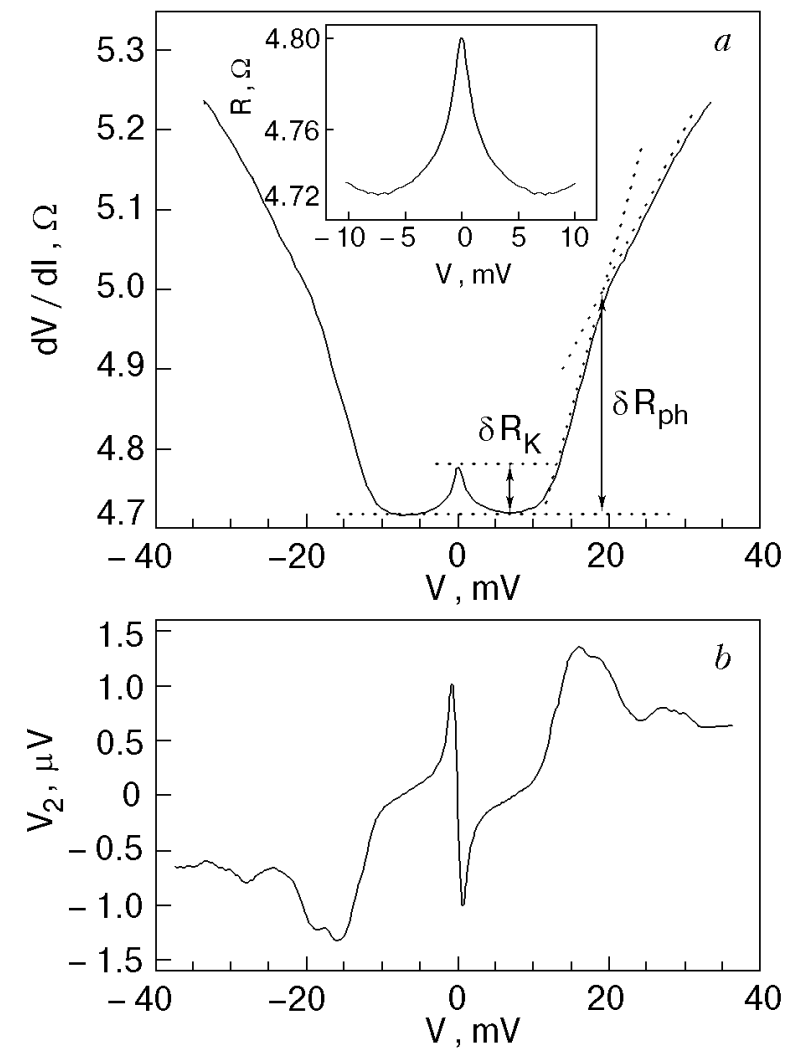

Fig. 1. Point contact spectrum for Kondo alloys of $\mathrm{CuCr}$ ( 0.1 at. \%). The first $V-I$ derivative $R=d V / d I$, where the arrows indicate the contribution to the differential resistance due to the interaction of conduction electrons with phonons $\delta R_{\mathrm{ph}}$, and Kondo impurities $\delta R_{K}$, inset: the Kondo peak in zero magnetic field on an expanded scale (a). The second $V-I$ derivative (b). The rms modulation voltage $V_{1}$ equals $585 \mu \mathrm{V}$. The rms second harmonic signal $V_{2}$ is connected with the second derivative via the equation $(1 / R)(d R / d V)=2^{3 / 2}\left(V_{2} / V_{1}^{2}\right) . T=1.6 \mathrm{~K}$, $H=0$. 
The inset illustrates the Kondo peak in zero magnetic field on an expanded scale. This peak in the PC spectrum of copper is accounted for by conduction electron scattering off the Kondo impurities of $\mathrm{Cr}$ in the vicinity of the point contact and is characterized by an increase of the differential resistance by the logarithmic law $d V / d I(V) \propto-\ln (V)$ at voltage biases lower than for the phonons for $\mathrm{Cu}$. The variations in the differential resistance due to the interaction between conduction electrons and the phonons, $\delta R_{p h}$, and the Kondo impurities, $\delta R_{K}$, are denoted by arrows. For the AuFe alloy, one can observe a similar phonon spectrum of $\mathrm{Au}$, while there is a Kondo peak of lower intensity in a lower bias range than for the phonons (not shown). The lower intensity of the Kondo peak compared to that in $\mathrm{CuCr}$ is due to the fact that the Kondo temperature in $\mathrm{AuFe}$ is one order of magnitude lower than in $\mathrm{CuCr}$.

It is known [8] that Kondo impurities such as $\mathrm{Cr}$ and $\mathrm{Fe}$ in copper form clusters at low concentrations, unlike the Mn impurity. Therefore, a portion of the samples (we measured more than 10 samples of $\mathrm{CuCr}$ alloy) exhibited a lower Kondo peak intensity for high diameters than those selected by us for detailed measurements. It should be emphasized that we studied all of the point contacts, even those whose lower peak intensities were indicative of clustering, and all of the samples display the size effect described below.

For pure metals, in the ballistic limit $d R / d V(V)$ is directly proportional to the electronphonon interaction (EPI) function $g_{P C}$ [9]

$$
\frac{1}{R} \frac{d R}{d V}(V)=\frac{8}{3} \frac{e d}{\hbar v_{F}} g_{P C}(e V) .
$$

The Sharvin resistance $R_{0}$ for zero bias is related to the $\mathrm{Cu}$ point contact diameter through the equation [10]:

$$
d \approx \frac{30}{\sqrt{R[\Omega]}}[\mathrm{nm}] .
$$

This equation is valid for contacts in the shape of a clean orifice. For Au point contacts the diameter was estimated by this equation with a nominator of 28 .

The essential parameter characterizing the PC spectrum is the maximum value of the EPI signal that determines the ballistic regime of electron transport through the point contact. The maximum value of the EPI signal for the point contacts was estimated by the relation which follows from (1) and (2) [9]:

$$
g_{P C}(e V)=\left(\frac{12.9}{\sqrt{R_{0}}}\right) \frac{d R}{d V},
$$

where the resistances $R_{0}$ and $R$ are expressed in ohms and the voltage $V$ in millivolts.

The estimate $g_{P C}^{\max }=0.24$ for $\mathrm{CuCr}$ is in good agreement with the value for point contacts of pure copper in the clean orifice model. The value of maximum EPI intensity for the AuFe point contacts, $g_{P C}^{\max }=0.1$, is also consistent with that for pure $\mathrm{Au}[9]$. Thus we are measuring point contacts with an electron transport regime close to the ballistic one.

\section{Contact-size dependence of phonon and Kondo scatterings}

A qualitative estimate of the electron-phonon interaction was made by determining the increase in the differential resistance from the minimum of the differential resistance for a given contact up to the resistance for a bias of about $20 \mathrm{mV}$ at the intersection of the interpolation straight lines $\left(\delta R_{\mathrm{ph}}\right)$ (Fig. 1). The estimate was performed with the assumption that the PC spectra of pure $\mathrm{Cu}$ exhibit no zero-bias anomalies. The interaction with the Kondo impurities was estimated by the peak amplitude for zero bias $\left(\delta R_{K}\right)$. The values of $\left(\delta R_{\mathrm{ph}}\right)$ and $\left(\delta R_{K}\right)$ for the AuFe alloy were determined in a similar way.

The relative differential resistance $\delta R_{\mathrm{ph}} / R_{0}$ and $\delta R_{K} / R_{0}$ versus the point contact diameter are shown in Fig. 2. $R_{0}$ is measured directly. The diameter is estimated from Eq. (2). The dashed line in the figure illustrates the diameter dependence of relative differential resistance associated with $\delta R_{\mathrm{ph}} / R_{0}$ for the clean orifice model [9]:

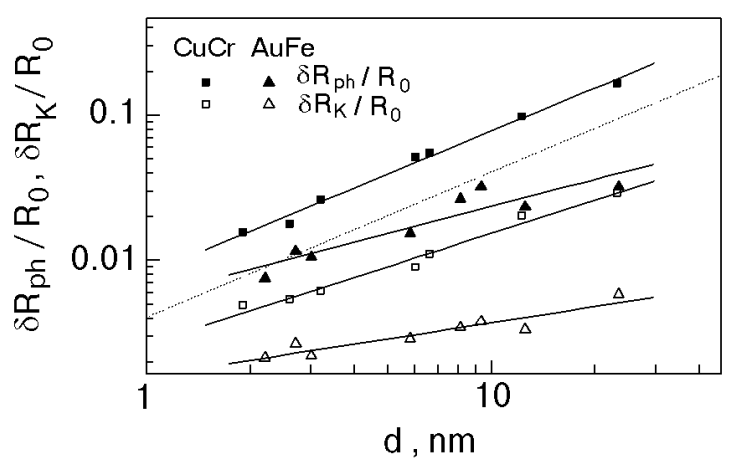

Fig. 2. Point-contact-diameter dependence of the relative variation of the differential resistance due to the conduction electron interaction with phonons and Kondo impurities for $\mathrm{CuCr}$ and $\mathrm{AuFe}$. (The dashed line represents Eq. (3)). Note the difference of slopes between the linear fits to the phonon and Kondo-impurities interactions shown as straight lines. 


$$
\begin{gathered}
\left(\frac{\delta R_{\mathrm{ph}}}{R_{0}}\right)_{V=20 m V}=\frac{8}{3} \frac{e d}{\hbar v_{F}} \int_{0}^{20 m V} g_{P C}(\omega) d \omega= \\
=4.05 \cdot 10^{-3} d[\mathrm{~nm}] .
\end{gathered}
$$

The discrepancy between the experimental PC diameter dependence of the EPI and the model dependence is accounted for by the difference in PC area geometry. We propose that this discrepancy is due to the fact that the production of the point contact by the break-junction technique involves a contact elongation accompanying a diameter reduction. Hence, it is necessary to introduce a parameter $L$ that characterizes the PC length in the Eq. (1) that gives linear interpolation between the theoretical limits for a clean orifice and a channel [11]:

$$
\frac{1}{R} \frac{d R}{d V}(V)=\frac{8}{3} \frac{e(d+3 \pi L / 4)}{\hbar v_{F}} g_{P C}(e V), \quad T \approx 0 .
$$

Here fluctuations of the local density of electron states at the Fermi energy in a channel-type point contact [3] result in an enhancement of electron scattering by Kondo impurities with decreasing the PC diameter. Linear fitting of the experimental PC diameter dependence of the EPI in logarithmic coordinates gives a slope of $0.98 \pm 0.04$. For $\mathrm{CuCr}$ the experimental curve thus obtained lies slightly above the same dependence for the orifice-type PC. Linear fitting of the points determining the electron scattering by the Kondo impurities gives a slope of $0.77 \pm 0.04$. The difference in the slopes of the Kondo scattering and the EPI implies an enhancement of the electron scattering by the Kondo impurities with decreasing PC diameter. As to the AuFe alloy, the experimental points of the EPI do not lie above the theoretical dependence. We consider this to be due to the fact that the impurity concentration for this alloy is at the boundary of changing over to a spin glass lying between the diffusive and ballistic regimes. The slopes of the EPI and the Kondo interaction linear fittings for AuFe are $0.62 \pm 0.10$ and $0.37 \pm 0.05$, respectively, also indicating the enhanced size effect.

\section{Energy dependence of Kondo scattering}

The next parameter directly related to a size effect in the Kondo temperature is the energy broadening of the zero-bias peak. In Fig. 3 (the lower inset) the dependence of the Kondo peak differential resistance is shown for two diameters. It is clearly seen that a decrease in the PC diameter (the diameters for the curves $d_{1}$ and $d_{2}$ are $12.2 \mathrm{~nm}$ and $1.9 \mathrm{~nm}$, respectively) causes the Kondo peak to become broader. We estimated the spectral broadening of the Kondo peak due to the experimental temperature and the modulation signal amplitude by the equation [12]:

$$
\delta(e V) \approx \sqrt{\left(3.53 k_{B} T\right)^{2}+\left(1.73 e V_{1,0}\right)^{2}}
$$

where $V_{1,0}$ is the amplitude of the modulation voltage across the point contact at zero bias. The calculated value of the spectral broadening, $\delta V \approx 0.86 \mathrm{mV}$ (indicated by the horizontal bar in the lower inset of Fig. 3), suggests that it does not result in significant instrumental broadening (though for the larger diameters the width of Kondo peak may suffer from experimental broadening). Note that the energy broadening of the Kondo peak is sensitive to the ballistic conditions of electron transport. The condition of ballistic electron transport $d \leq l_{i}, l_{e}$ depends on both the point contact preparation technology and contact area strain. The latter is due to multiple fracture and bringing the contact together repeatedly. The nature of the chemical element and concentration of magnetic impurities also have an effect.

Thus the peak broadening clearly observed with decreasing contact diameter is not related to the temperature and modulation smearing and is one more manifestation of a size effect. The upper inset in Fig. 3 illustrates the voltage characteristics of the same Kondo peaks on a semilogarithmic scale. It is evident that the energy broadening of the Kondo peak results in an increase in the slope of the logarithmic portion (the dashed line) which is in direct relation to the Kondo temperature. We believe now that the equations used in Ref. [2] to cal-

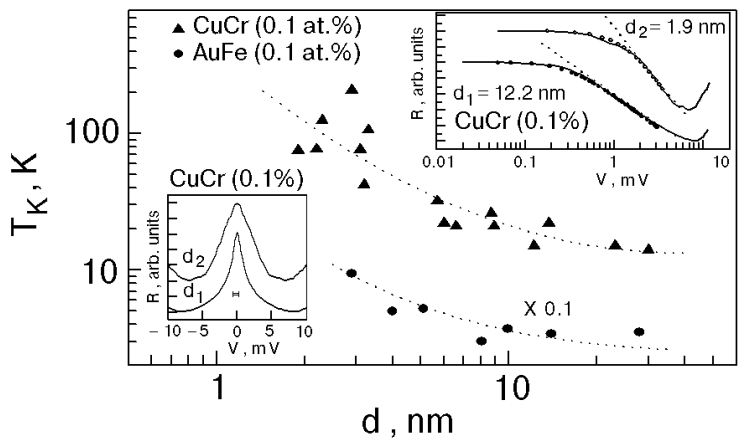

Fig. 3. Dependence of the Kondo temperature on the point contact diameter for $\mathrm{CuCr}$ and $\mathrm{AuFe}$ according to Eq. (4). Bottom inset: broadening of the Kondo peak $(\mathrm{CuCr})$ with decreasing contact diameter; the spectral resolution is shown as a horizontal bar; top inset: the Kondo peak of the same alloy with a logarithmic voltage scale $\left(d_{1}, d_{2}\right.$ are the diameters in both insets). For clarity, the points for AuFe alloy are presented a decade below. 
culate the Kondo temperature give a poor method of estimating it correctly from the PC characteristics. Their application for contacts of large diameter gave a Kondo temperature value different from that in bulk materials. Moreover, the Kondo temperature increases by several orders of magnitude as the contact diameter is reduced. Here we use instead, following Refs. [13], the empirical relation between the Kondo impurity resistivity in bulk alloys as a function of temperature [14]:

$$
\rho_{m}=A-B \ln \left[1+(T / \theta)^{2}\right] ;
$$

here $\ln \left(\theta / T_{K}\right)=-\pi[S(S+1)]^{1 / 2}$ where $S$ is the impurity spin, which we take equal to $1 / 2$ for both $\mathrm{Cr}$ and $\mathrm{Fe}$.

To compare the temperature dependence of the resistance in a bulk sample with the dependence of the Kondo scattering upon the applied voltage in a point contact, we translate the energy scales using the relation $V[\mathrm{mV}]=0.31 T[\mathrm{~K}]$ [15]. The Kondo temperature is estimated from fitting of the experimental dependence of the normalized Kondo peak by this formula, Eq. (4) (Fig. 3, upper inset). The dependence of the Kondo temperature obtained in this way upon the point contact diameter is shown in the Fig. 3. It is seen that for both alloys the Kondo temperature is considerably higher when the contact diameter is smaller. For large diameter contacts the Kondo temperature saturates at a value exceeding the corresponding bulk value. This discrepancy may arise because the empirical formula cannot properly treat the character of the Kondo scattering in ballistic point contacts, while it is fairly adequate for resistive measurement in bulk. Besides, the AuFe (0.1\%) alloy is in the spin-glass state at $T=1.6 \mathrm{~K}$ which leads to an apparent increase of $T_{K}$ determined by formula (4). Another reason for the different Kondo temperatures of a bulk sample and a larger-diameter point contact may be a deviation of the ballistic character of the electron transit through large diameters.

The enhanced intensity of the Kondo peak in the experiment may be attributed to the systematically increasing concentration of magnetic impurities in the point contact region, for example, due to mechanical deformation causing displacement of the impurities. Indeed, the higher the Kondo impurity concentration, the higher is the peak [1]. We might then expect that this uncontrollable growth of the Kondo-impurity content would lead, at certain concentration and quite low temperatures, to the formation of a spin-glass state, i.e., the splitting of the peak in the frozen internal magnetic field. In reality, however, when the contact diameter is de- creased, the peak splitting in external or internal magnetic fields (due to spin glass ordering at high nominal concentrations) is always suppressed.

\section{Size-effect of Kondo scattering in an external magnetic field}

The dependence of the magnetoresistance upon the point contact diameter is the third parameter manifesting the Kondo size effect in point contacts. In an external magnetic field the negative magnetoresistance is caused by the Zeeman effect [16], which restrains the spin degrees of freedom of the magnetic impurity and thus suppresses the Kondo effect. In our study the Kondo temperatures of the bulk $\mathrm{CuCr}$ and $\mathrm{AuFe}$ alloys are $2 \mathrm{~K}$ and $0.2 \mathrm{~K}$, respectively. They are within the interval where our maximal external magnetic field of $4.3 \mathrm{~T}$ at $T=1.6 \mathrm{~K}$ is unable to split the Kondo peak in $\mathrm{CuCr}$ but it can do this in AuFe. The size dependences $d V / d I(V)$ of the AuFe Kondo peak split in the field $4.3 \mathrm{~T}$ at $T=1.6 \mathrm{~K}$ are shown in Fig. $4, a$. This splitting is clearly seen for large-diameter contacts (Fig. 4, $a$, curves for $d=12$ and $10 \mathrm{~nm}$ ),
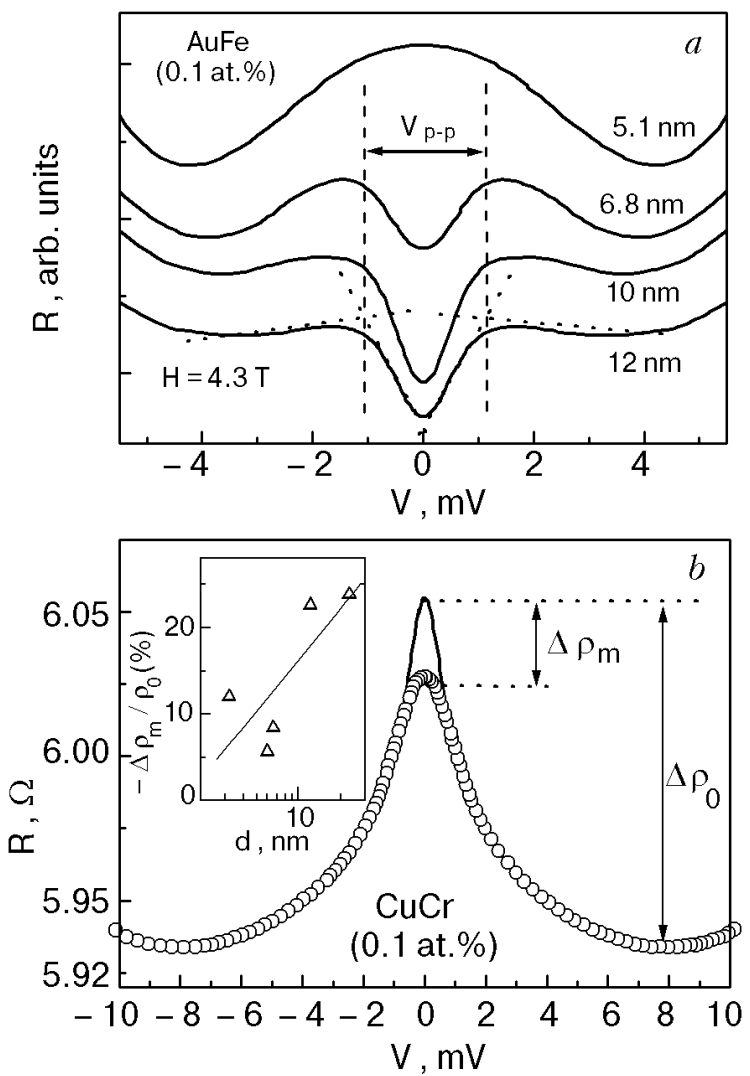

Fig. 4. Effect of an external magnetic field on the Kondo peak: An external field of $4.3 \mathrm{~T}$ splits the Kondo peak in AuFe alloys. The size effect results in suppression of splitting (curve with $d=5.1 \mathrm{~nm}$ ) (a); the Kondo peak in zero field (line) and at $4.3 \mathrm{~T}$ (circles) for $\mathrm{CuCr}$ alloys (the arrow shows the magnetoresistance) (b). Inset: the negative magnetoresistance at a field of $4.3 \mathrm{~T}$ as a function of the point contact diameter. 
where it is not influenced by the size effect. When the point contact diameter is decreased (curves for $d=6.8$ and $5.1 \mathrm{~nm}$ ), the splitting of the Kondo peak is suppressed though the position $V_{p-p}$ remains the same. The size effect enhances the interaction between the conduction electrons and the magnetic impurities. For small sizes it leads to the fact that the Zeeman energy $E=g \mu H S_{z}$ in a field $4.3 \mathrm{~T}$ is unable to fix the magnetic impurity spins with the field, i. e. , to restrict their degree of freedom and to exclude them from the Kondo interaction.

For the $\mathrm{CuCr}$ alloy the Kondo temperature $T_{K}=2 \mathrm{~K}$ and an external magnetic field $4.3 \mathrm{~T}$ is insufficient to split the peak. A similar result was obtain for $\mathrm{CuFe}$ [14] in a magnetic field of $12 \mathrm{~T}$ at $T=0.5 \mathrm{~K}$. The dependence $d V / d I(V)$ of the Kondo peak in zero field and $4.3 \mathrm{~T}$ are shown in Fig. $4, b$. The weaker intensity of the Kondo peak in the external magnetic field results from the negative magnetoresistance in these alloys due to the Zeeman energy (the magnetoresistance was taken as the differential resistance intensity of the Kondo peak at $V=0$ ). As follows from Fig. 3 of Ref. [17], the relative magnetoresistance $\left(-\Delta \rho_{M} / \rho_{0}\right)$ is directly proportional to the applied magnetic field and inversely proportional to temperature and the Kondo temperature. In our experiments the negative magnetoresistance decreases due to a decrease in the point contact diameter, with the external magnetic field and the temperature fixed. Hence, the change in magnetoresistance results from the rise of the Kondo temperature when the point contact diameter becomes smaller. The variation of the relative magnetoresistance $\left(-\Delta \rho_{M} / \rho_{0}\right)$ as a function at the point contact diameter is shown in Fig. $4, b$ (inset). It is seen that a decrease in the point contact diameter entails a considerable drop of the relative magnetoresistance. In our range of diameters the value of the drop coincides well with the increase in $T_{K}$ in the same diameter range ( $\mathrm{Fig} .3, \mathrm{CuCr}$ ). The size effect thus enhances the interaction between the conduction electrons and the Kondo impurities and leads to a drop of the negative magnetoresistance.

\section{Conclusions}

In summary we can infer the following conclusions.

The point-contact-spectroscopic studies of the Kondo size effect on the alloys $\operatorname{CuCr}(0.1$ at.\%) and $\mathrm{AuFe}(0.1$ at.\%) using the break-junction technique permit us to investigate the interaction of the conduction electrons with phonons and the Kondo impurity on the same contact of variable diameter. These studies have shown that:

- the interaction of conduction electrons with the Kondo impurities increases, relative to the EPI, with decreasing point contact diameter, similar to our earlier results on CuMn [2] and CuFe [13] alloys;

- a decrease in the point contact diameter leads to a rise of the Kondo temperature, which results in energy broadening of the peak;

- in the external magnetic field, a decrease in the point contact diameter causes a considerable drop of the negative magnetoresistance in the $\mathrm{CuCr}(0.1$ at.\%) alloy and suppresses the Kondo peak splitting in $\operatorname{AuFe}(0.1$ at.\%);

- in the $\mathrm{CuCr}$ alloy, saturation of the Kondo temperature is observed for large diameters.

1. A. A. Lysykh, I. K. Yanson, O. I. Shklyarevski, and Yu. G. Naidyuk, Solid State Commun. 35, 987 (1980); A. G. M. Jansen, A. P. van Gelder, P. Wyder, and S. Straessler, J. Phys. F11, L15 (1981); Yu. G. Naidyuk, O. I. Shklyarevskii, and I. K. Yanson, Fiz. Nizk. Temp. 8, 725 (1982) [Sov. J. Low Temp. Phys. 8, 362 (1982)].

2. I. K. Yanson, V. V. Fisun, R. Hesper, A. V. Khotkevich, J. M. Krans, J. A. Mydosh, and J. M. van Ruitenbeek, (a) Phys. Rev. Lett. 74, 302 (1995), (b) Fiz. Nizk. Temp. 20, 1062 (1994) [Low Temp. Phys. 20, 836 (1994)].

3. G. Zarand and L. Udvardi, Phys. Rev. B54, 7606 (1996).

4.J. F. DiTusa, K. Lin, M. Park, M. S. Isaacson, and J. M. Parpia, Phys. Rev. Lett. 68, 678 (1992).

5. G. Chen and N. Giordano, Phys. Rev. Lett. 66, 209 (1998); C. Strunk, M. Henny, and C. Schönenberger, Phys. Rev. Lett. 81, 2982 (1998).

6. V. Chandrasekhar, P. Santhaman, N. A. Penebe, R. A. Webb, H. Vloeberghs, C. Van Haesendonck, and Y. Bruynseraede, Phys. Rev. Lett. 72, 2053 (1994).

7. C. J. Muller, J. M. van Ruitenbeek, and L. J. de Jongh, Physica C191, 485 (1992).

8. M. Hansen, Constitution of Binary Alloys, McGraw-Hill, New York (1958).

9. A. V. Khotkevich and I. K. Yanson, Atlas of Point Contact Spectra of Electron-Phonon Interactions in Metals, Kluwer Academic Publ. (1995).

10.Yu. V. Sharvin, Zh. Eksp. Teor. Fiz. 48, 984 (1965) [Sov. Phys. JETP 21, 655 (1965)].

11.I. K. Yanson, V. V. Fisun, R. Hesper, J. M. Krans, J. A. Mydosh, N. van der Post, and J. M. van Ruitenbeek, Physica B218, 77 (1996).

12. A. M. Duif, Doctraalsriptie, KUN Nijmegen, The Netherlands (1983).

13. N. van der Post, F. Mettes, J. A. Mydosh, J. M. van Ruitenbeek, and I. K. Yanson, Fiz. Nizk. Temp. 22, 313 (1996) [Low Temp. Phys. 22, 245 (1996)]; Phys. Rev. B53, R476 (1996).

14. M. D. Daybell, in: Magnetism, G. Rado and H. Suhl (eds.), Academic Press, New York (1973) Vol. 5, p. 121.

15. B. I. Verkin, I. K. Yanson, I. O. Kulik, O. I. Shklyarevskii, A. A. Lysykh, and Yu. G. Naidyuk, Solid State Commun. 30, 215 (1981).

16. P. Monod and S. Schultz, Phys. Rev. 173, 645 (1968).

17. M. D. Daybell and W. A. Steyert, Phys. Rev. Lett. 20, 195 (1968). 